Original Article

Bikram Shakya, M. Ch.

Department of Neurological Surgery, KMCTH

Bidur KC, M. Ch.

Department of Neurological Surgery, KMCTH

Amit Thapa, M. Ch.

Professor, Department of Neurological Surgery, KMCTH

Address for correspondence:

Dr. Bikram Shakya

Department of Neurological Surgery, KMCTH.

Mobile No. 98512383265,

E-mail: drsbikram@gmail.com

Date submitted : 28 June 2019

Date accepted : 31 August 2019

$\mathrm{U}$ p to 500,000 surgeries in the U.S. has been complicated by Surgical site infections (SSIs). ${ }^{1,9}$ This accounts for 1 million excess days of hospitalization and \$1.6 billion in additional healthcare expenditure. ${ }^{17,22}$

Intravenous prophylactic antibiotics have systemic distribution, which may limit bactericidal concentrations locally, with the risk of systemic adverse effects, including hypersensitivity reactions and renal toxicity. ${ }^{4}$ Topical antibiotics, by contrast, have the advantage of achieving
Nepal Journal of Neurosciences 16:20-24, 2019

\section{Topical use of Vancomycin in Craniotomy}

In neurosurgery, surgical site infections are major cause of morbidity and mortality. It has been a significant burden in clinical practice, despite of best practices with or without use of antibiotics. Topical antibiotics are one potential method reducing the problem.In our study, we seek to evaluate the benefits of topical vancomycin. In this Institutional Review Board-approved retrospective observational study, patients who underwent neurosurgical intervention in Department of Neurological Surgery KMCTH enrolled retrospectively from October 1, 2014 to September 30, 2018. Patients in whom topical vancomycin powder applied intraoperatively for postoperative SSI prophylaxis and those without, comprising the vast majority of neurosurgical patients within the KMCTH, were examined. Patients presenting with infection, open wound, multiple scheduled surgeries, application of topical antibiotics other than vancomycin, or multiple antibiotics were excluded. Patients data were analyzed within the first 30 days after surgery.There were total 259 patients with 190 as control and 69 as cases. With all these surgeries undergone we had SSI in 5, 1.93\% ( $\mathrm{p}=<0.001)$ of emergency surgeries, where Vancomycin powder was not used. There were no surgical site infection in any of the cases where topical Vancomycin powder was used.Topicalvancomycin is a safe, effective, and economical measure to prevent SSIs following craniotomy.

Key words: Craniotomy, Surgical site infection, Vancomycin

high local concentrations at the surgical site, where contaminating pathogens are located, while minimizing systemic toxicity. Due to the increasing prevalence of nosocomial methicillin-resistant Staphylococcus aureus (MRSA), which now accounts for as many as half of all SSIs, ${ }^{25}$ efficacy of topical vancomycin for reducing SSIs is being increasingly explored. ${ }^{18}$

In our study, we seek to evaluate the benefits of topical vancomycin. We hypothesize that application of topical vancomycin during closure of craniotomy reduces the 
incidence of SSIs postoperatively. There is currently not much data assessing the efficacy of topical vancomycin among neurosurgical patients undergoing craniotomy, and a prospective, randomized clinical trial is needed. With this study we could identify, justify and thus eliminate important post-operative morbidity, the SSI in neurosurgery.

\section{Materials and Method:}

In this Institutional Review Board-approved study, patients who underwent neurosurgical intervention in Department of Neurological Surgery KMCTH were enrolled retrospectively from October 1, 2014 to September 30, 2018.

Patients were divided into two groups, with and without topical application of vancomycin powder. We used 1 gm of Vancomycin powder sprinkled equally all over the surgical area prior to closure of skin. Patients in whom topical vancomycin powder applied intraoperatively for postoperative SSI prophylaxis and those without (No Vancomycin group, NVG), comprising the vast majority of neurosurgical patients within the KMCTH, were examined. Patients presenting with infection, open wound, multiple scheduled surgeries, application of topical antibiotics other than vancomycin, or multiple antibiotics; were excluded. Data related to pre and post-operative antibiotic administration, surgical service, surgery scheduling, and postoperative infection were collected from the electronic and other medical record.

Data registration were done in the designated Performa, prepared for the study. Registration of baseline and clinical data were collected starting on admission in the hospital. Patients presenting in ER and NS OPD, who had been admitted and underwent neurosurgical intervention as routine and emergency surgery were identified and needful details collected.

Data was collected using a pre-designed questionnaire. These collected data were entered in SPSS data sheet, subjected for descriptive statistics, diagrams and the needful z test. Statistical analysis was done using SPSS computer software version 15.0. The $\mathrm{p}$ value of $<0.05$ was taken as statistically significant.

\section{Results:}

There were total 259 patients with 190 as control and 69 as cases. The age ranges from 18 to 63 years with mean age of presentation being 40 years. Altogether 209 Males outnumbered 50 females. The most of the surgeries undergone were emergency $(n=167)$ with elective surgery being 92 .

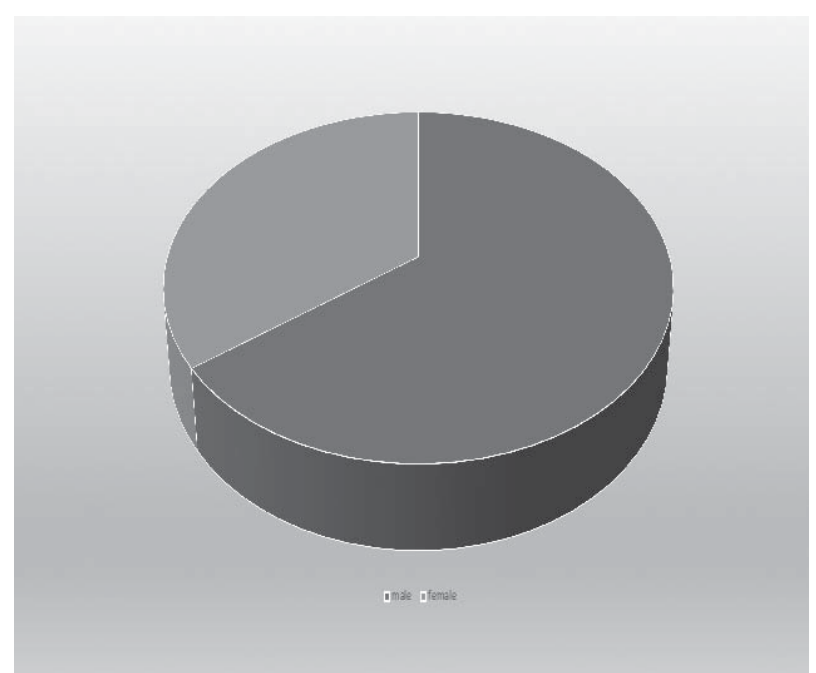

Figure 1. Gender Distribution

Among surgeries undertaken, they could be categorized as Trauma $76 \% n(n=196)$, vascular $15 \%(n=39)$, tumor $7 \%(\mathrm{n}=18)$ and miscellaneous $18(2 \%)$.

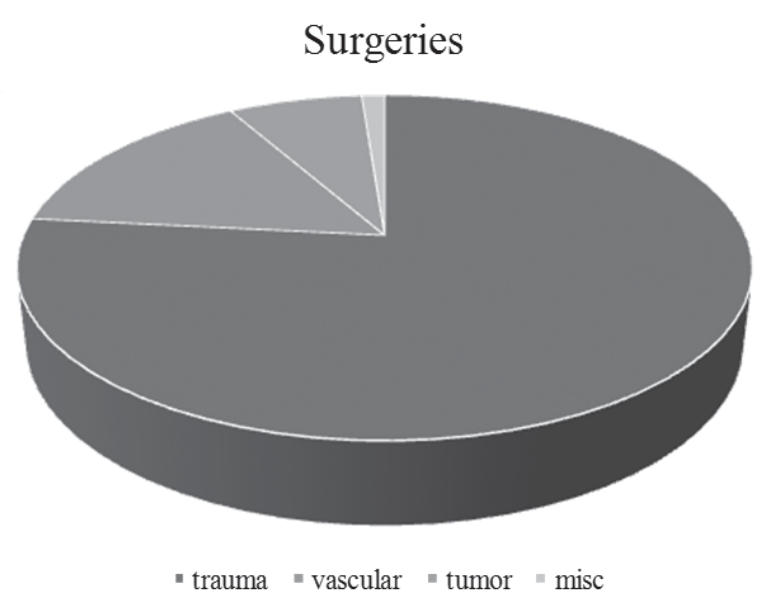

Figure 2. Type of surgeries

With all these surgeries undergone we had SSI in 5, $1.93 \%$ ( $\mathrm{p}=<0.001$ )of emergency surgeries, where Vancomycin powder was not used. There were no surgical site infection in any of the cases where topical Vancomycin powder was used. Among the infected cultures there were mixed growth of $E$. coli and Acinetobacter in 3 (5.6\%) cases, causing deep infection. One patient (2.8\%) developed meningitis. One was only radiological diagnosis without any growth seen on culture (Fig. 3).Treatment wasinitiated with local wound care and broad spectrum intra venous antibiotics till swab culture and antibiogram results were finalized.Definite treatment was done with removal of free bone flaps completing the course of needful intravenous antibiotics followed by prolonged period of oral antibiotics. One of the patients didn't agree 


\section{Shakya et al}

to proceed with further management and left hospital against medical advice. With this study known side effects associated with parenteral administration of vancomycin did not appear to occur when the drug is applied topically into a surgical wound.

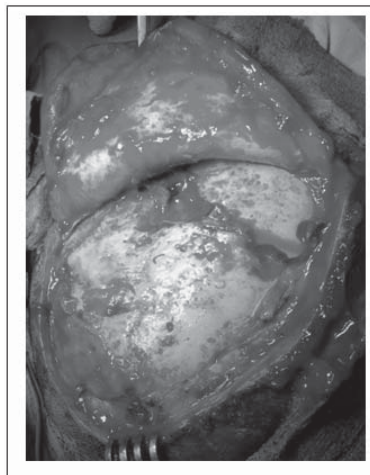

(a)

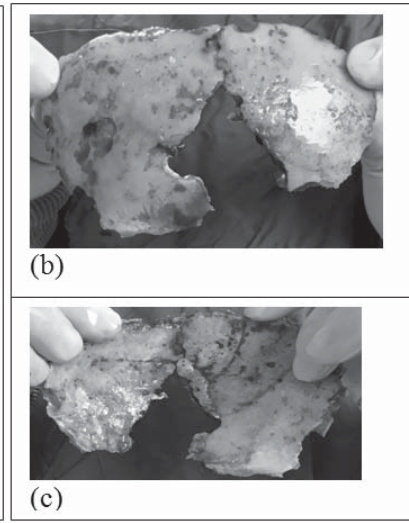

Figure 3: Surgical site infection: (a)Intraoperative osteomyelitic bone with pathological fracture and bone resorption, (b) superior view of the skull bone removed, (c) inferior view of the skull bone removed.

\section{Discussion:}

"Certainly infections cannot be attributed to the intervention of the devil but must be laid at the surgeon's door” Cushing ${ }^{16}$

Surgical site infections (SSIs) is nightmare for every surgeon including neurosurgeons. It has been a significant burden in clinical practice, despite of best practices with or without use of antibiotics. Vancomycin powder has been applied to wounds easily, is inexpensive, and can achieve high local concentrations with low systemic levels. ${ }^{10,3}$

SSIs after craniotomy complicate $2-5 \%$ of approximately 2 million procedures annually, ${ }^{20}$ triple the length of stay (LOS), and on average cost an additional $\$ 26,000$ per case. As per published studies, incidence of SSIs in neurosurgery overall varies from 1 to $11 \%{ }^{2}$ Several types of wound infections can appear after a brain surgery including meningitis, epidural abscess, subdural empyema, brain abscess, and bone flap osteomyelitis. ${ }^{8}$ In this study, we present the first Nepali large institutional series of open craniotomy patients treated with topical vancomycin with the goal of preventing postoperative SSI.

There has been many investigations with protective beneficial effect of intrawound vancomycin in both instrumented and non-instrumented spine surgeries. ${ }^{6,11,18,23}$ It is important to note that topical vancomycin prevents S. aureus infection, the leading cause of SSIs. Ideally, these antibiotics would achieve minimal systemic absorption, a high local concentration, and have minimal local and systemic adverse event. In this study, among the infected cultures there were mixed growth of $E$. coli and Acinetobacter in 5.6\%cases, causing deep infection.A similar benefit was demonstrated in cardiothoracic surgery when vancomycin paste was applied to the cut edges of the sternum during sternotomy closure. ${ }^{24}$

In a recent large meta-analysis, risk factors associated with SSI following open craniotomies were identified and included other infection, number of previous operations, CSF leak, CSF drainage, duration of operation, venous sinus entry, ASA (American Society of Anesthesiologists) score $>2$, sex (male), and nontraumatic surgical indications. ${ }^{14}$ Though we studied some but not all of these variables.

The finding of our study was found to have significant protective effect of topical vancomycin against SSI. With all surgeries undergone we had SSI in 1.93\% ( $p=<0.001)$ of emergency surgeries, where Vancomycin powder was not used. There were no surgical site infection in any of the cases where topical Vancomycin powder was used.This corelates with the study findings of McCutcheon BA et al, ${ }^{7}$ where they found significant protection against SSIs (OR: 0.33; CI: 0.18-0.60), with the use of vancomycin powder after cranial operation. Similarly Chang SM et $\mathrm{al}^{5}$ pointed out statistically significant reduction in SSIs from $5 \%$ to $1.4 \%$ after cranial surgeries. Those studies pointed out these findings to be novel without having been previously investigated or described by other metanalysis. However with limited number of surgeries, our rate of SSIs in NVG, $2.6 \%$, along with the studies by afore mentioned authors in NVG is relatively high compared to current literature.

The costs of SSI are high in terms of both morbidity and hospital expense. Deep cranial wound infections may compromise the bone flap, dura, and brain and necessitate additional surgeries and prolonged antibiotic use. In severe cases, SSI may lead to abscess and death. If we calculate in financial prospect, definitely there has been reduction in total health care cost as there has been reduction in hospital stay. Beyond the direct costs of treatment, lost wages and economic productivity compound the financial impact. Also there has been no financialburden to the patient for further management of infection. Ravikumar $\mathrm{V}$ et $\mathrm{al}^{5}$ in their study approved this fact that use of Vancomycin being associated with reduction of healthcare costs. In this way, this intervention may also provide indirect financial gains from increased reimbursement with lower SSI rates.

Being retrospective study, there definitely is need of prospective study with inclusion of more number of cases. In this respect, this study is similar to large number of spinal studies that examined vancomycin efficacy.,12,13,21 
Similarly, this study could have been specific if there has been correlation of the final outcome to the comorbidities that patient had, indication of the surgeries, diagnosis, etc. Here we couldn't deny the possibility that patient-related factors, indications and diagnosis, may influence infection risk. With prospective study we could definitely be able to compare the total cost that a patient has benefitted by not having, thus preventable SSI. The decision on using intrawound vancomycin was based on the surgeon's preference and was not decided in prior. This is a multisurgeon single-center study; the rate of SSI and type of organisms might differ based on the geographical location, surgeons practice, and patient-specific factors.

Additionally, this study utilized a surgery-centered analysis, in which each surgery was analyzed as a single event regardless of whether a patient underwent multiple surgeries. This approach was taken since utilization of topical vancomycin was dependent on each individual surgery and not each individual patient.Similarly, here is always a chance of unreported infections or complications from patients being seen at outside institutions. However, findings of this present study has encouraged us to proceed with further study to proceed prospectively, thus including detail variables like length of hospital stay ,duration of surgery, confounding factors that could have led to infection, etc.

\section{Conclusion:}

This study has given the positive information for vancomycin use in cranial surgery thus significantly decreasing SSIs. There has been need of more prospective, larger, randomized, longer follow-up studies for more reliable result. There is insufficient evidence to suggest that routine single-use vancomycin powder leads to antibiotic resistance. Routine use of vancomycin powder as a surgical adjunct is supported by the available literature and warrants further examination in randomized controlled trials. We hypothesize that topical vancomycin use reduces infection rates by directly affecting any local inoculum at the time of surgery. A randomized prospective trial is warranted.

\section{References}

1. Yasunaga H, Ide H, Imamura T, Ohe K. Accuracy of economic studies on surgical site infection. J Hosp Infect 65 (2): 102-107, 2007

2. Walcott BP, Neal JB, Sa Sheth, et al. The incidence of complications in elective cranial neurosurgery associated with dural closure material. J Neurosurg 120: 278-284, 2014
3. Sweet FA, Roh M, Sliva C. Intrawound application of vancomycin for prophylaxis in instrumented thoracolumbar fusions: efficacy, drug levels, and patient outcomes. Spine (Phila Pa 1976) 36: 20842088, 2011

4. Stone HH. Basic principles in the use of prophylactic antibiotics. J Antimicrob Chemother 14 Suppl B: 33-7, 1984

5. Ravikumar V, Ho AL, Pendharkar AV, Sussman ES, Kwonghon Chow K, Li G. The use of vancomycin powder for surgical prophylaxis following craniotomy. Neurosurgery 80 (5): 754-758, 2017

6. O’Neill KR, Smith JG, Abtahi AM, et al. Reduced surgical site infections in patients undergoing posterior spinal stabilization of traumatic injuries using vancomycin powder. Spine J 11 (7): 641-646, 2011

7. McCutcheon BA, Ubl DS, Babu M, et al. Predictors of surgical site infection following craniotomy for intracranial neoplasms: an analysis of prospectively collected data in the American college of surgeons national surgical quality improvement program database. World Neurosurg88: 350-358, 2016

8. McClelland S, 3rd, Hall WA. Postoperative central nervous system infection: incidence and associated factors in 2111 neurosurgical procedures. Clin Infect Dis 45 (1): 55-59, 2007

9. LissovoyG. de, FraemanK., Hutchins V., Murphy D., Song D., Vaughn B.B. Surgical site infection: incidence and impact on hospital utilization and treatment costs. Am J Infect Control 37: 387-97, 2009

10. Lazar HL, Barlam T, Cabral H. The effect of topical vancomycin ap- plied to sternotomy incisions on postoperative serum vancomycin levels. J Card Surg 26: 461-5, 2011

11. Khan NR, Thompson CJ, DeCuypere M, et al. A meta-analysis of spinal surgical site infection and vancomycin powder. J Neurosurg Spine 21 (6): 974-983, 2014

12. Hill BW, Emohare O, Song B, Davis R, Kang MM. The use of vancomycin powder reduces surgical reoperation in posterior instrumented and noninstrumented spinal surgery. ActaNeuro chir (Wien) 156: 749-754, 2014

13. Heller A, McIff TE, Lai SM, Burton DC. Intrawound vancomycin powder decreases staphylococcal surgical site infections following posterior instrumented spinal arthrodesis. J Spinal Disord Tech: E584-E589,2013

14. Fang C, Zhu T, Zhang P, Xia L, Sun C. Risk factors of neuro- surgical site infection after craniotomy: A 


\section{Shakya et al}

systematic review and meta-analysis. Am J Infect Control 45: e123-e134, 2017

15. Devin CJ, Chotai S, McGirt MJ, et al. Intrawound vancomycin decreases the risk of surgical site infection after posterior spine surgery: A multicenter analysis. Spine 43 (1):65-71, 2015

16. Cushing. Concerning the results of operations for brain tumor. JAMA 64:189-195, 1915

17. Chiang HY, Kamath AS, Pottinger JM, et al. Risk factors and outcomes associated with surgical site infections after craniotomy or craniectomy. J Neurosurg 120: 509-52, 2014

18. Chiang HY, Herwaldt LA, Blevins AE, Cho E, Schweizer ML. Effectiveness of local vancomycin powder to decrease surgical site infections: a metaanalysis. Spine J 14 (3): 397-407, 2014

19. Chang SM, Parney IF, McDermott $M$, et al. Perioperative complications and neurological outcomes of first and second craniotomies among patients enrolled in the Glioma Outcome Project. J Neurosurg 98 (6): 1175-118, 2003

20. ChaichanaKL, BydonM, Santiago-DieppaDR, et al. Risk of infection following posterior instrumented lumbar fusion for degenerative spine disease in 817 consecutive cases. J Neurosurg Spine 20 (1): 4552, 2014
21. Caroom C, Tullar JM, Benton EG Jr, Jones JR, Chaput CD: Intrawoundvancomycin powder reduces surgical site infections in posterior cervical fusion. Spine (Phila Pa 1876) 38:1183-1187, 2013

22. Buchanan CC, Hernandez EA, Anderson JM, et al. Analysis of 30-day readmissions among neurosurgical patients: surgical complication avoidance as key to quality improvement. J Neurosurg 121: 170-175, 2014

23. Bakhsheshian J, Dahdaleh NS, Lam SK, Savage JW, Smith ZA. The use of vancomycin powder in modern spine surgery: systematic review and meta-analysis of the clinical evidence. World Neurosurg 83 (5): 816-823, 2015

24. ArrudaMV, BraileDM, Joaquim MR, Suzuki FA, Alves RH. The use of the vancomycin paste for sternal hemostasis and mediastinitis prophylaxis. Rev Bras Cir. Cardiovasc 23 (1): 35-39, 2008

25. Anderson DJ, Sexton DJ, KanafaniZA, AutenG, Kaye KS. Severe surgical site infection in community hospitals: epidemiology, key procedures, and the changing prevalence of methicillin-resistant Staphylococcus aureus. Infect Control Hosp Epidemiol 28 (9): 1047-53, 2007 